\title{
La vinculación con el medio en la Universidad de Santiago de Chile. El alcance territorial de los proyectos financiados entre 2018 y 2020
}

\author{
Diego Salazar Alvarado \\ Coordinador Fondo VIME. \\ Universidad de Santiago de Chile. \\ diego.salazar.a@usach.cl \\ (iD) orcid.org/0000-0001-7362-930X
}

RECEPCIÓN: 26/06/20

ACEPTACIÓN FINAL: 11/09/20

\section{Resumen}

El presente trabajo describe el alcance

territorial de las acciones de vinculación con el medio de la Universidad de Santiago de Chile en la Región Metropolitana desde su programa de financiamiento de proyectos. Para ello se emplea la información obtenida a partir de los proyectos financiados por el Fondo VIME entre el año 2018 y 2020. De los resultados, se identifica una mayor presencia de relaciones en el entorno inmediato a la universidad y una menor presencia en lugares que presentan un mayor índice de prioridad social. El trabajo propone algunos elementos a considerar a la hora de medir o evaluar la función universitaria estudiada, además de abrir el debate en torno a la relación entre la universidad y el contexto social actual.

Palabras clave: vinculación con el medio; extensión universitaria; alcance territorial; prioridad social; Fondo VIME
Sujetos y relaciones en extensión universitaria / Desafíos de gestión

다(1)(5) (2)
Public engagement in the University of Santiago de Chile. The territorial scope of the projects financed between 2018 and 2020

\section{Abstract}

This paper describes the territorial scope of the Universidad de Santiago de Chile's outreach actions in the Metropolitan Region, from its project funding program. It uses information obtained from projects financed by the VIME Fund between 2018 and 2020. From the results, a greater presence of relations in the immediate surroundings of the university and with less presence in places with a higher index of social priority is identified. The work proposes some elements to be considered when measuring or evaluating the university function studied, in addition to opening the debate on the relationship between the university and the current social context.

Keywords: public engagement; university extension; territory; social priority; Fondo VIME
Vinculação com o meio na Universidade de Santiago do Chile. O escopo territorial dos projetos financiados entre 2018 e 2020

\section{Resumo}

Este documento descreve o escopo territorial das ações de vinculação com o meio da Universidade de Santiago do Chile na Região Metropolitana, a partir de seu programa de financiamento de projetos. Para este fim, são utilizadas informações obtidas de projetos financiados pelo Fundo VIME entre 2018 e 2020. A partir dos resultados, identifica-se uma maior presença de relações no entorno imediato da universidade e com menor presença em lugares que apresentam um índice maior de prioridade social. O trabalho propõe alguns elementos a serem considerados ao medir ou avaliar a função universitária estudada, além de abrir o debate sobre a relação entre a universidade e o contexto social atual.

Palavras-chave: vinculação com o meio extensão universitária; âmbito territorial; prioridade social; Fundo VIME

Para citación de este artículo: Salazar Alvarado, D. (2020). La vinculación con el medio en la Universidad de Santiago de Chile. El alcance territorial de los proyectos financiados entre 2018 y 2020. +E: Revista de Extensión Universitaria, 10(13), e0017. doi: 10.14409/extension.2020.13.Jul-Dic.e0017 


\section{Introducción}

El sistema educativo chileno cuenta con el legado de las políticas neoliberales y privatizadoras de la dictadura cívico-militar. Su carácter segregador y de profunda mercantilización sigue vigente pese a las reformas impulsadas por los últimos gobiernos como respuesta a las movilizaciones sociales (Bellei, 2020; Orellana et al., 2019).

La educación superior se ha expandido en Chile de forma muy acelerada y guiada por los vaivenes del mercado. Según Orellana (2016), hay al menos tres promesas implícitas e incumplidas en dicha expansión: las de igualdad, de libertad y democracia, y de desarrollo. El autor concluye en su trabajo que la responsabilidad de la universidad pública es pensar la construcción de una educación superior para el país asumiendo dichas promesas incumplidas. Para ello, la universidad pública estaría obligada a abrir sus puertas a la sociedad (Orellana, 2016). Paradójicamente, la función universitaria encargada de la relación universidad-sociedad no es un tema que convoque preocupación en los movimientos estudiantiles, en las comisiones políticas de educación, ni en la literatura científica. En particular, existe un reducido desarrollo del área en nuestro país.

Esta función universitaria ha generado un interés creciente en torno a las necesidades de conceptualización y evaluación de la vinculación, principalmente desde las mismas casas de estudios, respondiendo a los requerimientos de la Comisión Nacional de Acreditación. ${ }^{1}$

Para la vinculación o la extensión universitaria, el territorio "aparece como el 'terreno de juego', allí donde la teoría se vuelve práctica” (Arzeno, 2018, p. 4), es donde se generan las dinámicas de intercambio y creación de conocimiento mediante el diálogo o confrontación de saberes (Sousa Santos, 2007). En dichos territorios, la universidad, en tanto fenómeno urbano, también se hace parte como una de las fuerzas que se relacionan social y espacialmente, interactuando y negociando según sus intereses (Addie, 2019). Para las universidades estatales, la función de vinculación con el medio apunta principalmente a identificar problemas de los actores sociales y contribuir a llegar a los mecanismos y modos que apuntalen el desarrollo territorial, dimensión que va más allá del puro desarrollo económico (Programa de las Naciones Unidas para el Desarrollo -PNUD—, 2018). Según lo anterior, se comprende que las dinámicas que se desarrollan en los territorios son de variada complejidad. En tanto, el presente artículo se limita a definir al territorio únicamente en su carácter geoespacial, comprendiendo que esta es solo una de las dimensiones que lo constituyen.

Así, este artículo tiene como objetivo describir la vinculación con el medio desarrollada por la Universidad de Santiago de Chile (USACH) en la Región Metropolitana, lugar en el que se encuentra emplazada la institución. Para ello, se emplean los datos de 61 proyectos llevados a cabo en el marco del Fondo a la Vinculación con el Medio (VIME) entre los años 2018 y 2020.

Se presenta aquí la idea de vinculación con el medio en Chile, muy ligada a las definiciones generadas por la Comisión Nacional de Acreditación, para luego dar paso a la exposición de los elementos centrales de la política institucional de vinculación de la USACH. Más adelante se comentan las categorías a trabajar y se realiza el cruce de los datos obtenidos. Finalmente, con el análisis realizado según las variables anteriormente planteadas, se elaboran las conclusiones y se proponen elementos a considerar para la evaluación y medición

1) Con objeto de impulsar la calidad en las instituciones de educación superior, el Consejo Nacional de Acreditación supervisa los estándares que deben cumplir las universidades en su quehacer. 
de la función universitaria estudiada, además de abrir el debate en torno a qué universidad necesita la sociedad en el contexto de efervescencia social en Chile y de pandemia.

\section{Vinculación con el medio y extensión universitaria}

La misión universitaria de vinculación con el medio o de extensión es un campo de gran heterogeneidad y diversidad a la hora de concretarse en acciones. Tiene un carácter polisémico y ambiguo en sus definiciones, es un significante en disputa (González et al., 2017; Tommasino \& Cano, 2016).

Junto a ello, es caracterizada como una función postergada, de tercer orden, y su centralidad está en la docencia y la investigación. En palabras de Oscar Jara:

"Pese a la reconocida y trillada afirmación de que esta es una dimensión sustantiva del quehacer universitario, al igual que la investigación y la docencia, el caso es que no es valorada de igual manera y continúa siendo muchas veces considerada como un área o una dimensión de menor importancia e incluso prescindible". (Jara, 2019, p. 4)

En ese marco, las funciones universitarias "se encuentran inconexas entre sí" (Tommasino \& Cano, 2016, p. 11). Como respuesta, se ha desarrollado la idea de la integralidad de las funciones universitarias; y para avanzar en dicha dirección, valor fundamental cobra la curricularización de la extensión, combinación de enseñanza, investigación y extensión (Adán et al., 2016, p. 38).

Si bien el objetivo del texto no es realizar una discusión conceptual respecto del término, es necesario comprender que en Chile se desarrolla para la función universitaria extramuros el concepto de vinculación con el medio, desde la cual se busca sostener relaciones bidireccionales con actores extrauniversitarios, es decir, con una perspectiva dialógica, de comunicación horizontal y de "intercambio y sinergias, convergencias o eventuales confrontaciones" (Fleet et al., 2017, p. 14). Se desarrolla dicha conceptualización con objeto de superar "la extensión como relación vertical y paternalista" (Adán et al., 2016). De todas formas, algunas universidades entienden a la extensión tradicional como una de las acciones incluidas en el quehacer de la vinculación con el medio (Dougnac, 2016; PNUD, 2018), lo que indica que es una definición aún en construcción y, por lo tanto, tensionada y en discusión.

El concepto se desarrolla al alero de la Comisión Nacional de Acreditación (Adán et al., 2016, p. 42). No obstante, el artículo 17 de la Ley 20129, que establece un Sistema Nacional de Aseguramiento de la Calidad de la Educación Superior, define como optativa la acreditación en vinculación con el medio para los planteles. La ley plantea que, para conseguir esta acreditación,

"la institución de educación superior debe contar con políticas y mecanismos sistemáticos de vinculación bidireccional con su entorno significativo local, nacional e internacional, y con otras instituciones de educación superior, que aseguren resultados de calidad. Asimismo, deberán incorporarse mecanismos de evaluación de la pertinencia e impacto de las acciones ejecutadas, e indicadores que reflejen los aportes de la institución al desarrollo sustentable de la región y del país". 
Si bien, según el portal web de la Comisión Nacional de Acreditación, al momento en que se escriben estas líneas existen 40 universidades acreditadas en vinculación con el medio, las observaciones sobre los déficits en medición y criterios para identificar impacto son de una amplia presencia.

\section{La vinculación con el medio desde la USACH}

La USACH es heredera de una tradición extensionista muy presente a lo largo de su historia, desde sus orígenes en la Escuela de Artes y Oficios hasta nuestros días, aspecto que se profundizó con la fundación, en 1947, de la Universidad Técnica del Estado (UTE), institución ligada al desarrollo del país con una agenda de extensión cultural y de trabajos voluntarios como un sello de esta. Enrique Kirberg, rector de la UTE, elegido democráticamente por académicos, funcionarios y estudiantes en el marco de la reforma universitaria de finales de la década de 1960, plantea que la extensión universitaria adquiere otro rol de cara al proceso reformista:

"No se puede concebir ahora tal labor sino como una promoción cultural que sea ventana abierta directamente al pueblo, a través de la cual se entregue ciencia, técnica, arte, a fin de sembrar más y más inquietudes de progreso y de incorporación plena a la vida". (Kirberg, 1970, p.190)

Dicho proceso, que contó con figuras como Víctor Jara en la Secretaría de Extensión, fue truncado y atacado materialmente por la dictadura militar. ${ }^{2}$

La USACH nace de la división de la UTE, decretada por la dictadura militar. Particularmente, la USACH se asienta en la otrora casa central de la UTE, que contaba con sedes en distintas partes del país.

Con la recuperación de la democracia, el quehacer extensionista siguió siendo parte de las actividades universitarias. Sin embargo, el principal hito de institucionalización sucedió con la fundación en 2012 de la Vicerrectoría de Vinculación con el Medio. La actual Política de Vinculación con el Medio de la Universidad de Santiago nació de un proceso participativo desarrollado en 2018 junto a académicos, funcionarios y estudiantes. En ella se define la vinculación con el medio como:

"Una responsabilidad y un deber de la Universidad de Santiago de Chile como entidad estatal y pública, pilar fundamental para la investigación y docencia, que le permite asegurar su compromiso y contribución permanente al desarrollo integral de la sociedad". (Universidad de Santiago de Chile, 2018, p.6)

Ello se concretaría en acciones y relaciones que pueden "variar en sus grados de formalización, tiempo y nivel de bidireccionalidad". Además, estas conllevarían elementos de "docencia, investigación, extensión y del quehacer general de la Universidad" (Universidad de Santiago de Chile, 2018, p. 6). Los principios que guían dichas acciones serían "la de-

2) La UTE fue atacada por militares y tanquetas el 11 de septiembre de 1973, en el marco del golpe de Estado al gobierno de Salvador Allende. Con la instalación de los militares en el gobierno, fue designado un coronel como rector, el que comenzó un proceso de desarticulación y persecución de la comunidad universitaria. 
mocratización del conocimiento y la cultura, el respeto e integración, la responsabilidad y sustentabilidad, la bidireccionalidad y la ética" (Vicerrectoría de Vinculación con el Medio, 2019, p. 4). En definitiva, la política de vinculación con el medio es de un carácter general y opera como orientación de los planes, programas y actividades que desarrollen las distintas unidades académicas de la Universidad.

Junto a lo anterior, en los documentos institucionales se reconoce a la vinculación "como un área esencial y transversal al cumplimiento de la misión institucional, lo que se plasma con claridad en el Plan Estratégico Institucional 2016-2020" (Vicerrectoría de Vinculación con el Medio, 2019, p. 3). El carácter de transversalidad de la función se puede relacionar con la idea de integralidad desarrollada en la discusión Latinoamericana en cuanto a la extensión crítica.

Con la finalidad de fomentar la vinculación con el medio en la comunidad académica se promoverá "la creación de sistemas que permitan contar con los recursos económicos y humanos necesarios para realizar actividades y programas de vinculación" (Universidad de Santiago de Chile, 2018, p. 15). Siguiendo dicha línea de acción, la Vicerrectoría de Vinculación con el Medio creó el Fondo VIME, fuente de financiamiento de proyectos que tiene como objetivo:

"Promover, fortalecer y consolidar las actividades de vinculación con el medio desarrolladas por las unidades académicas y la comunidad universitaria a través del financiamiento y apoyo a iniciativas de docencia, investigación, extensión y prestación de servicios que involucren la participación de actores del medio". (Vicerrectoría de Vinculación con el Medio, 2019, p. 4)

Se establecen así cuatro áreas de desarrollo para los proyectos, a saber: docencia con sello de vinculación con el medio, investigación con sello de vinculación con el medio, comunicación del conocimiento, y prestación de servicios.

\section{Metodología}

Para la caracterización del alcance territorial de los proyectos de extensión financiados por el Fondo VIME se empleó la información de los documentos administrativos del período 2018-2020. Dicha información se encuentra sistematizada en una base de datos de la Unidad de Estudios e Instrumentos de la Vicerrectoría de Vinculación con el Medio. De los datos obtenidos se realizó un análisis estadístico descriptivo (Blalock, 1998) que relacionó el sector al que pertenecen los actores, definido según su quehacer o actividad, el área de ejecución o tipo de proyecto, y la distribución territorial de los actores en la Región Metropolitana. La información fue trabajada en el entorno de desarrollo Rstudio en su versión 4.0.1, un software que trabaja con el lenguaje de programación $\mathrm{R}$ empleado para la relación de análisis estadísticos. Es distribuido bajo licencia GNU GPL, es decir, es un software libre, gratuito y de código abierto. ${ }^{3}$ 


\section{Categorías por sector $y$ territorio}

Los actores se encuentran categorizados en cuatro sectores: educación, productivo, público y social. Dichas categorías son construidas según las actividades que desarrolla cada actor participante.

- Educación: son establecimientos del sistema educativo escolar. Sistema de naturaleza mixta, es decir, existen instituciones de propiedad del Estado y privadas. Los establecimientos pueden ser: particulares pagados: establecimientos privados que cobran mensualidad; particulares subvencionados: establecimientos privados que reciben aportes del Estado con el objeto de reducir o eliminar cobros a las familias de estudiantes; municipales: establecimientos estatales y gratuitos, con dependencia municipal; corporaciones de administración delegada: establecimientos de educación media técnico-profesional fiscales, administrados por instituciones del sector público, personas jurídicas no lucrativas, que su quehacer tenga relación directa con las finalidades del establecimiento educacional.

- Productivo: actores que tienen participación en la productividad de la región o el país. Incluye: pequeñas y medianas empresas. Cooperativas: asociaciones que tienen por objeto mejorar las condiciones de vida de sus socios mediante la distribución de del excedente entre los mismos; trabajadores independientes: emprendimientos individuales, familiares $u$ asociaciones de microemprendedores.

- Público: instituciones dependientes del Estado. Incluye: gobiernos locales, servicios públicos, instituciones públicas autónomas, ministerios.

- Social: organizaciones de la sociedad civil. Incluye: organizaciones comunitarias, gremios, sociedades científicas, organizaciones no gubernamentales, deportivas, religiosas de base.

Los proyectos que se desarrollan son heterogéneos en sus objetivos, actividades y actores del medio que participan. El Fondo VIME, en sus bases de postulación, distingue cuatro áreas de desarrollo de los proyectos:

- Docencia: proyectos de docencia de pregrado, posgrado y educación continua, ligados a las mallas curriculares, que se desarrollan en relación con actores del medio con la finalidad de formar con perspectiva de responsabilidad social. Comprenden prácticas profesionales, asignaturas con la metodología de aprendizaje más servicio, entre otros. Abarcan temas como salud comunitaria en distintos territorios, memoria histórica y local, diálogo de saberes indígenas en torno a la astronomía, desarrollo de redes comunitarias para la gestión territorial, ingeniería en construcción en comunidades escolares, manejo de alimentos con pequeños productores, etcétera.

- Investigación: proyectos que crean conocimiento mediante procesos investigativos en vinculo colaborativo con actores extrauniversitarios, los que pueden participar en toda la investigación o en parte de esta. Comprenden iniciativas de investigación-acción, ciencia ciudadana, sistematización de experiencias, etc. Se han desarrollado en torno a la igualdad de género en escuelas, salud afectiva y sexual en comunidades, derecho a la vivienda con organizaciones sociales, salud mental en escuelas públicas, patrimonio cultural en antiguos enclaves mineros, medio ambiente y calidad del aire con organizaciones no gubernamentales, rendición de cuentas y transparencia con instituciones del Estado, etcétera.

- Comunicación: son proyectos relacionados con la difusión, divulgación y generación 
de espacios de intercambio de conocimiento que ponen en valor los diferentes tipos de saberes y formas de conocimiento presentes en la sociedad. Pueden ser actividades como encuentros, ferias y talleres. Se han desarrollado actividades como las ferias de matemáticas con escuelas, torneos deportivos paraolímpicos, encuentros de arte comunitario, talleres abiertos de enfermería y primeros auxilios, generación de medios de comunicación comunitarios, piezas de divulgación del conocimiento en filosofía.

- Servicios: los proyectos de esta área buscan solucionar problemas, mejorar las condiciones de vida y del entorno mediante el conocimiento desarrollado en la universidad; además incluyen la difusión de conocimiento para soluciones técnicas, más asociado a dinámicas unidireccionales en el modo de relacionarse con actores del medio, tales como operativos de salud en comunidades carentes y adulto mayor, fomento a la lectura, teatro educativo, formación para pequeños productores, etcétera.

\section{Categorías territoriales}

A nivel territorial se emplean dos categorizaciones de las comunas de la Región Metropolitana: por un lado, según el criterio de distancia del único campus universitario de la institución, se establecieron arbitrariamente cuatro niveles de distancia, inspirándonos en la teoría de los círculos concéntricos de Ernest Burgess (Park \& Burgess, 1925), que busca explicar los procesos de expansión de las ciudades mediante la representación gráfica de una serie de círculos que designan zonas sucesivas de asentamiento, diferenciándolas y enumerándolas desde el centro hacia afuera.

Para efectos de esta investigación, el centro no sería el distrito comercial central definido por Burgess sino que estaría representado por la USACH y ubicado en la comuna de Estación Central. Desde dicho punto se sucederían los siguientes círculos: las tres primeras zonas serían comunas que se encuentran dentro de la ciudad de Santiago, tales como entorno inmediato, comunas intermedias y comunas distantes. Por otro lado, las comunas de la zona más lejana serían todas las comunas pertenecientes a la Región Metropolitana que no se encuentran dentro de la ciudad. Las zonas definidas son:

- Entorno inmediato: incluye la comuna en la que se encuentra el campus y las comunas colindantes. Comprende Cerrillos, Estación Central, Lo Prado, Pedro Aguirre Cerda, Quinta Normal y Santiago. Aunque son colindantes a Estación Central, no se incluyen las comunas de Maipú y Pudahuel debido a su gran tamaño.

- Comunas intermedias: las inmediatamente colindantes a la categoría Entorno inmediato. Incluyen Cerro Navia, Conchalí, Independencia, La Cisterna, La Granja, Lo Espejo, Macul, Maipú, Ñuñoa, Providencia, Pudahuel, Recoleta, Renca, San Joaquín, San Miguel y San Ramón.

- Comunas distantes: aquellas colindantes a las Comunas intermedias pero solo considerando a las que son parte de la ciudad de Santiago. Comprenden El Bosque, Huechuraba, La Florida, La Pintana, La Reina, Las Condes, Peñalolén, Puente Alto, Quilicura, San Bernardo y Vitacura.

- Fuera de la ciudad: aquellas que son parte de la Región Metropolitana y que se encuentran fuera de la ciudad de Santiago. Consideran a Alhué, Buin, Calera de Tango, Colina, Curacaví, El Monte, Isla de Maipo, Lampa, Lo Barnechea, María Pinto, Melipilla, Padre Hurtado, Paine, Peñaflor, Pirque, San José de Maipo, San Pedro, Talagante y Tiltil. 
En la Figura 1 se puede apreciar el mapa con los cuatro niveles de distancia, según la teoría de los círculos concéntricos de Ernest Burgess.

Figura 1: Comunas de la Región Metropolitana según distancia del campus universitario

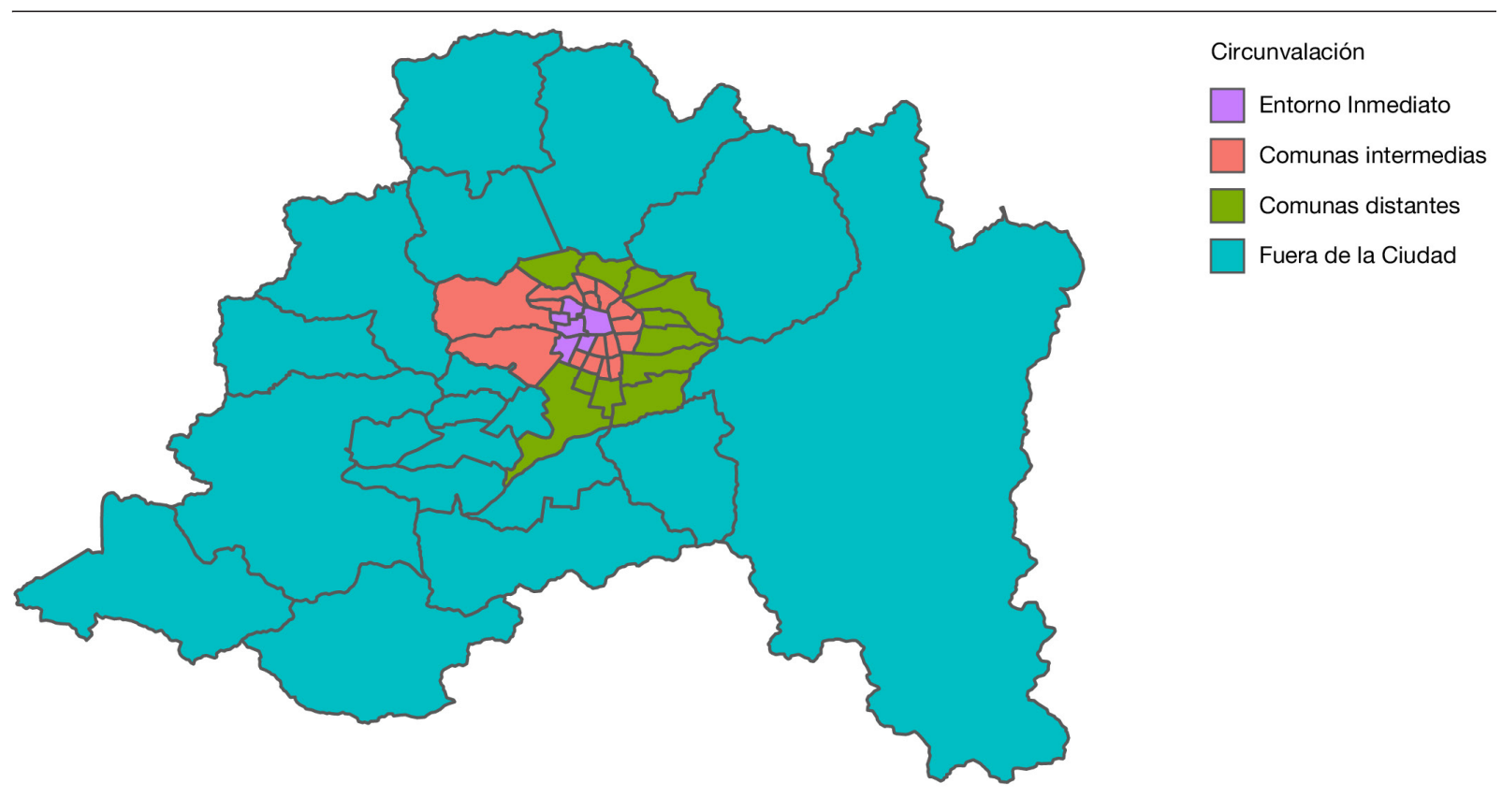

Fuente: elaboración propia desde los datos del Fondo VIME-USACH

Por otra parte, se utiliza el Índice de Prioridad Social (IPS), instrumento de medición desarrollado por el Ministerio de Desarrollo Social, para medir "el desarrollo socioeconómico alcanzado -en un momento determinado- por la población de las comunas de la Región Metropolitana de Santiago" (Ministerio de Desarrollo Social y Familia, 2019, p. 4). EI IPS contempla las dimensiones de ingreso, educación y salud. La dimensión de ingreso se incluye considerando los indicadores generados desde el porcentaje de población comunal perteneciente al $40 \%$ de menores ingresos de la calificación socioeconómica del Ministerio de Desarrollo Social y Familia y por el ingreso promedio de afiliados al Seguro de Cesantía. Para la dimensión de educación se emplean los resultados de pruebas estandarizadas en educación básica, los resultados de Prueba de selección universitaria y el porcentaje de reprobación en enseñanza media. Finalmente, la dimensión salud se define según la tasa de años de vida potencialmente perdidos por la muerte prematura de habitantes de entre 0 y 80 años, la de fecundidad específica de mujeres de entre 15 y 19 años, y el porcentaje de niños y niñas menores de 6 años en situación de malnutrición.

Con un procedimiento de estandarización de indicadores y agregación de estos se genera una escala común de 0 a 100; el valor máximo representa una situación más prioritaria y el valor inferior la situación menos prioritaria. De dicha escala se agrupan las siguientes categorías según priorización:

- Alta prioridad: Cerro Navia, Isla de Maipo, La Pintana, Lo Espejo, María Pinto y San Ramón. 
- Media alta prioridad: Buin, Conchalí, Curacaví, El Bosque, El Monte, Lo Prado, Melipilla, Padre Hurtado, Paine, Recoleta, San Bernardo, San Joaquín y San José de Maipo.

- Media baja prioridad: Alhué, Estación Central, Independencia, La Cisterna, La Granja, Lampa, Pedro Aguirre Cerda, Peñaflor, Pirque, Pudahuel, Quinta Normal, Renca, San Pedro, Talagante y Tiltil.

- Baja prioridad: Calera de Tango, Cerrillos, Colina, Huechuraba, La Florida, Macul, Maipú, Peñalolén, Puente Alto, Quilicura, San Miguel y Santiago.

- Sin prioridad: La Reina, Las Condes, Lo Barnechea, Ñuñoa, Providencia y Vitacura. ${ }^{4}$

En la Figura 2 se observa el mapa con las comunas identificadas conforme a los agrupamientos definidos desde el IPS.

Figura 2: Comunas de la Región Metropolitana según Índice de Prioridad Social

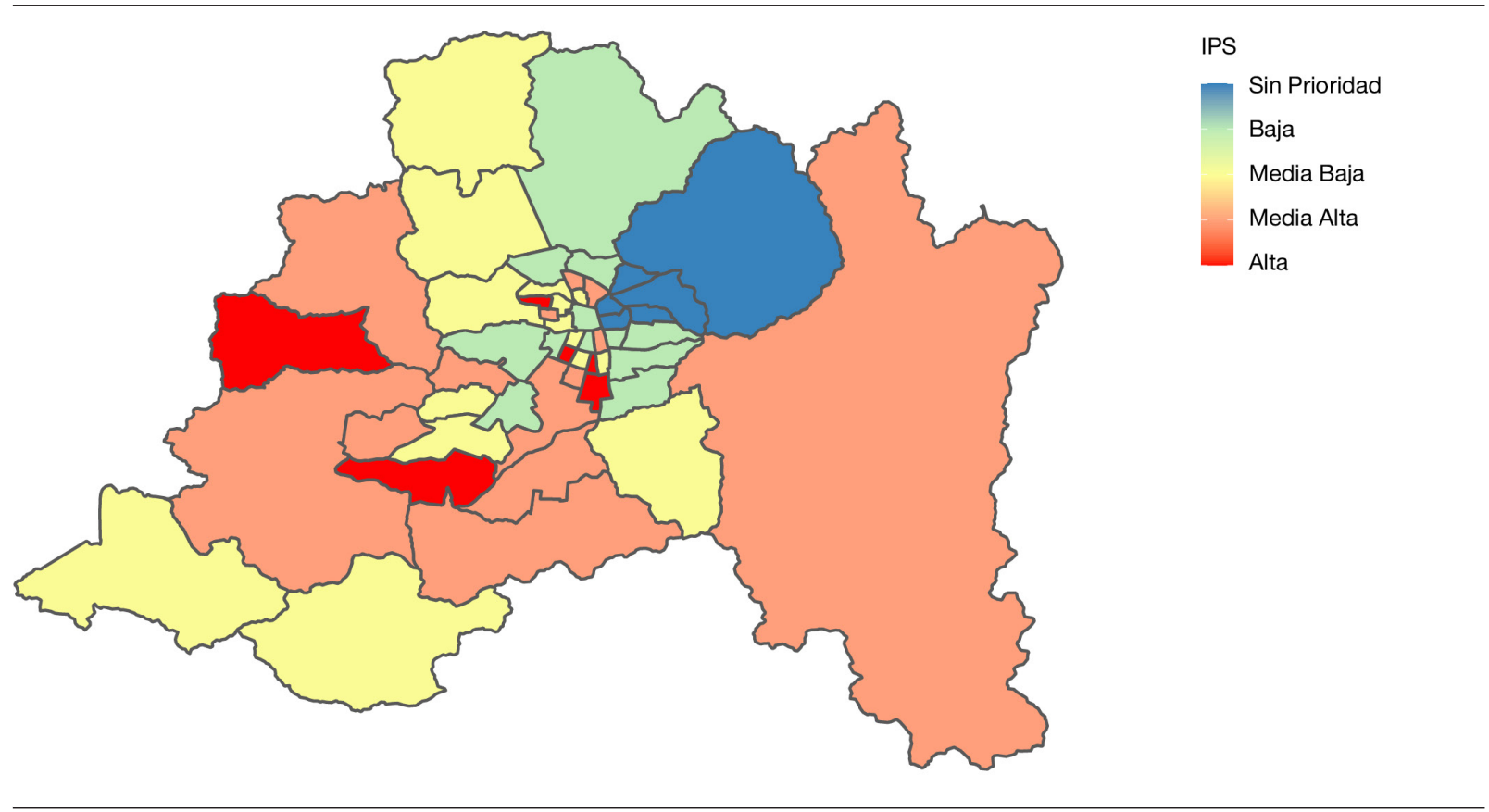

Fuente: elaboración propia según las categorías del Ministerio de Desarrollo Social y Familia (2019)

\section{Presentación de resultados}

En este apartado se presentan los resultados en tres subapartados según sector del actor, proximidad al campus de este, y el índice de prioridad social de la comuna en la que se desenvuelve. 


\section{Actores según sector}

Los proyectos considerados involucraron en sus actividades a 112 actores extrauniversitarios. En la Tabla 1 se muestra la distribución de actores según sector.

\begin{tabular}{|c|c|c|}
\hline Sector & Cantidad & $\%$ \\
\hline Educación & 49 & 43,8 \\
\hline Productivo & 19 & 17,0 \\
\hline Público & 15 & 13,4 \\
\hline Social & 29 & 25,9 \\
\hline Total & 112 & 100,0 \\
\hline
\end{tabular}

Fuente: elaboración propia.

Asimismo, en esta Tabla 1 se identifica que la mayor cantidad de actores es del sector Educación. Se generaron relaciones con 21 establecimientos particulares subvencionados, 18 municipales, 7 particulares pagados, y 3 de administración delegada. En cuanto al sector Social, 11 son organizaciones comunitarias, 8 corresponden a organizaciones no gubernamentales, 8 son deportivas, 1 es sociedad científica, y 1 es una organización religiosa de base. Del sector Productivo, 12 son pequeñas empresas (dedicadas a la innovación en construcción, salud, impresión 3D, entre otras actividades), 6 cooperativas (principalmente ligadas al comercio de productos agrícolas), y 1 asociación de trabajadores independientes (del sector comercial y el turismo). Finalmente, del sector Público, 9 son gobiernos locales, 4 servicios públicos de salud y educación, 1 ministerio del gobierno central, y 1 institución pública autónoma. En la Figura 3 se presenta la distribución de actores según área de proyecto.

Los datos dejan ver que existe una gran cantidad de actividades de comunicación, como ferias, talleres y eventos para actores de Educación y del sector Social con participación del $43,8 \%$ de los actores extrauniversitarios. Dichas actividades se sustentan principalmente en el diálogo, la divulgación de las ciencias en las comunidades y el encuentro con otros tipos de saberes construidos por los actores, como el saber experiencial, popular o ancestral.

Además, se visualiza un porcentaje no menor de las actividades de docencia e investigación, ya que ambas concentran el 35,8 \% de la participación. Lo anterior cobra relevancia porque tanto los proyectos del área de docencia como los del área de investigación, a efectos del Fondo VIME, apuntan a la curricularización y a la consolidación de la integralidad de las funciones universitarias. Los primeros deben responder a alguna asignatura reconocida en la malla curricular de la o las carreras involucradas, mientas que los segundos generan investigaciones formales que involucran a estudiantes, académicos, funcionarios universitarios, junto a actores extrauniversitarios. En la Tabla 2 se presentan en detalle los datos. 
Figura 3: Porcentaje de actores extrauniversitarios distribuidos según sector y área de proyecto

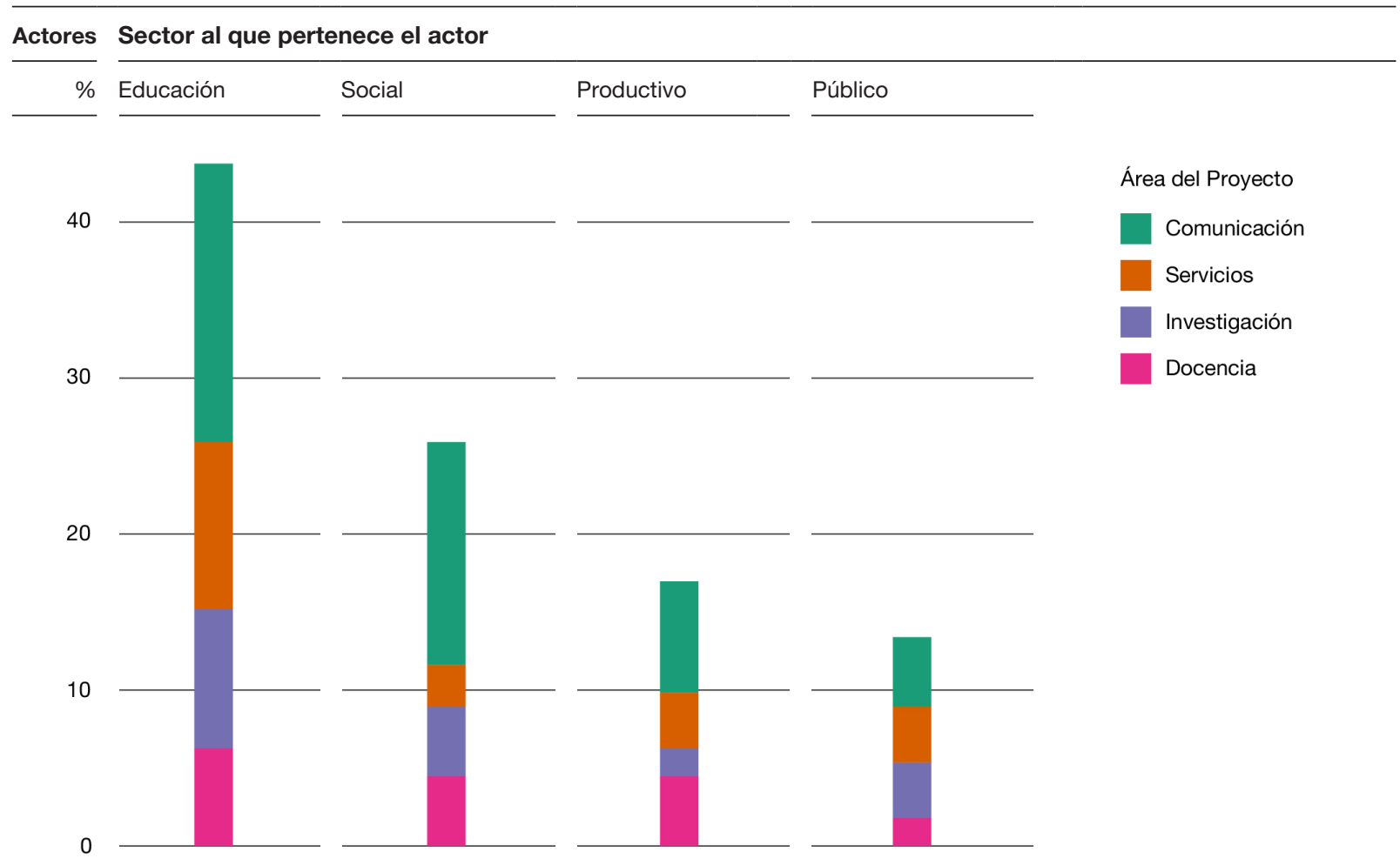

Fuente: elaboración propia desde los datos del Fondo VIME-USACH

Tabla 2: Distribución porcentual de actores extrauniversitarios según sector y área de proyecto

\begin{tabular}{|c|c|c|c|c|c|}
\hline Sector & Comunicación & Docencia & Investigación & Servicios & Total \\
\hline Educación & $17,9 \%$ & $6,2 \%$ & $8,9 \%$ & $10,7 \%$ & $43,8 \%$ \\
\hline Productivo & $7,1 \%$ & $4,5 \%$ & $1,8 \%$ & $3,6 \%$ & $17,0 \%$ \\
\hline Público & $4,5 \%$ & $1,8 \%$ & $3,6 \%$ & $3,6 \%$ & $13,4 \%$ \\
\hline Social & $14,3 \%$ & $4,5 \%$ & $4,5 \%$ & $2,7 \%$ & $25,9 \%$ \\
\hline Total & $43,8 \%$ & $17,0 \%$ & $18,8 \%$ & $20,5 \%$ & $100,0 \%$ \\
\hline
\end{tabular}

Fuente: elaboración propia.

\section{Actores según proximidad al campus}

En relación con la distribución territorial, es importante destacar que el $47,3 \%$ de los actores extrauniversitarios está ubicado en el entorno inmediato. En las comunas intermedias, el $28,6 \%$; en las comunas distantes, el $17 \%$; y fuera de la ciudad, el 7,1 \%. Notoriamente, en la medida en que la ubicación es más lejana al campus universitario menos actores participan. La Figura 4 muestra la presencia a nivel comunal de los actores del medio participantes en proyectos del Fondo VIME. 


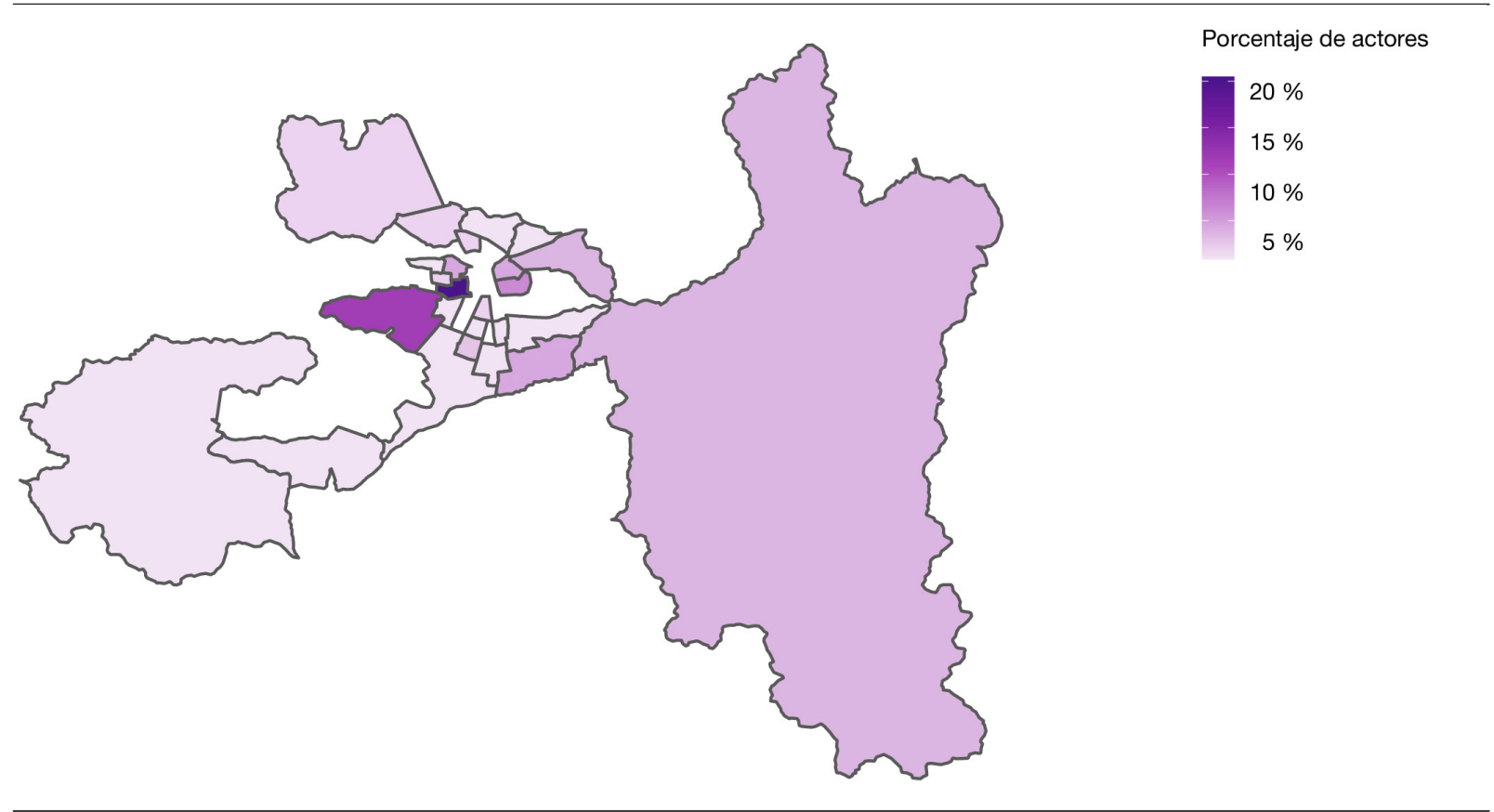

Fuente: elaboración propia desde los datos del Fondo VIME-USACH

En esta figura también se visualiza que existe una presencia importante de actores participantes de los proyectos desarrollados en la Región Metropolitana, lo que equivale a 26 comunas de un total de 52, es decir, el $50 \%$ de estas. En la Figura 5 se presenta la distribución de los actores extrauniversitarios según la categorización territorial anteriormente planteada.

Si bien existe presencia en varias comunas de la región, estas se concentran principalmente en el entorno inmediato, trabajado en particular con actores del sector de Educación y Social. En la Tabla 3 se exponen los porcentajes en detalle.

En todos los sectores, la mayoría de los actores se encuentra ubicada en el entorno inmediato. Respecto de las comunas distantes, podemos identificar que tienen una mayor proporción de actores en el sector Público, aunque sigue siendo menor en relación con el sector educación y social. Otro elemento para considerar es que hay muy pocos actores que se encuentran fuera de la ciudad de Santiago. 
Figura 5: Porcentaje de actores según sector y distancia del campus

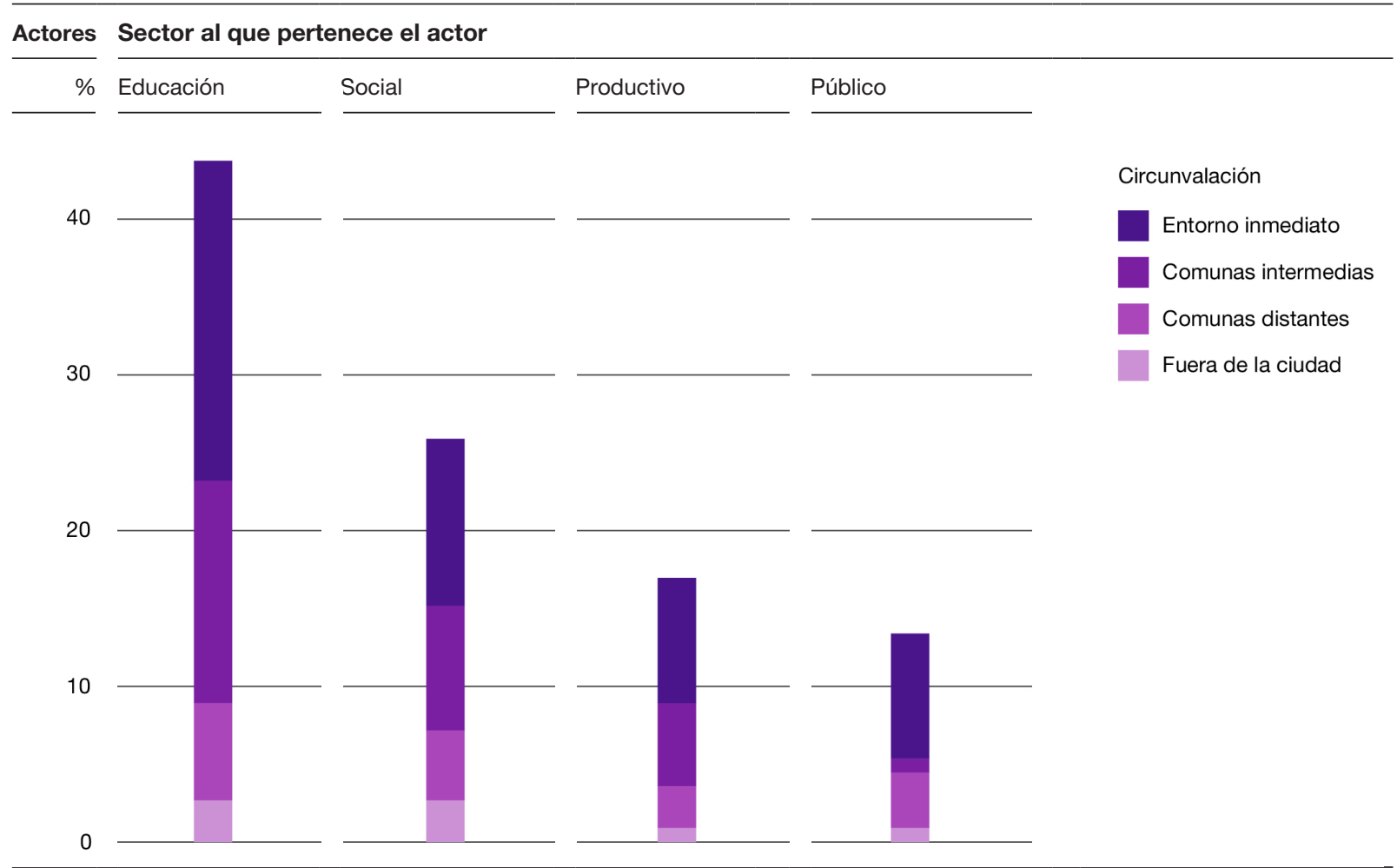

Fuente: elaboración propia desde los datos del Fondo VIME-USACH

Tabla 3: Porcentaje de actores extrauniversitarios según distancia del campus de actores del medio por sector

\begin{tabular}{|c|c|c|c|c|c|}
\hline Sector & $\begin{array}{r}\text { Entorno } \\
\text { inmediato }\end{array}$ & $\begin{array}{r}\text { Comunas } \\
\text { intermedias }\end{array}$ & $\begin{array}{l}\text { Comunas } \\
\text { distantes }\end{array}$ & $\begin{array}{r}\text { Fuera de la } \\
\text { ciudad }\end{array}$ & Total \\
\hline Educación & $20,5 \%$ & $14,3 \%$ & $6,2 \%$ & $2,7 \%$ & $43,8 \%$ \\
\hline Productivo & $8,0 \%$ & $5,4 \%$ & $2,7 \%$ & $0,9 \%$ & $17,0 \%$ \\
\hline Público & $8,0 \%$ & $0,9 \%$ & $3,6 \%$ & $0,9 \%$ & $13,4 \%$ \\
\hline Social & $10,7 \%$ & $8,0 \%$ & $4,5 \%$ & $2,7 \%$ & $25,9 \%$ \\
\hline Total & $47,3 \%$ & $28,6 \%$ & $17,0 \%$ & $7,1 \%$ & $100,0 \%$ \\
\hline
\end{tabular}

Fuente: elaboración propia.

Además, los actores son en gran medida de las comunas más próximas al campus, y concentran entre estas comunas el $61 \%$ de las organizaciones con las que existen proyectos. En la Figura 6 se presentan las cuatro comunas con mayor concentración. Se destaca que entre Estación Central y Santiago suman un $41 \%$ de los actores. Por otro lado, hay una gran diferencia entre la cantidad de actores de las tres primeras comunas (Estación Central, Santiago y Maipú), del $12 \%$ de la tercera pasa a un 6 \% en Ñuñoa. 
Figura 6: Comunas con mayor cantidad de actores del medio

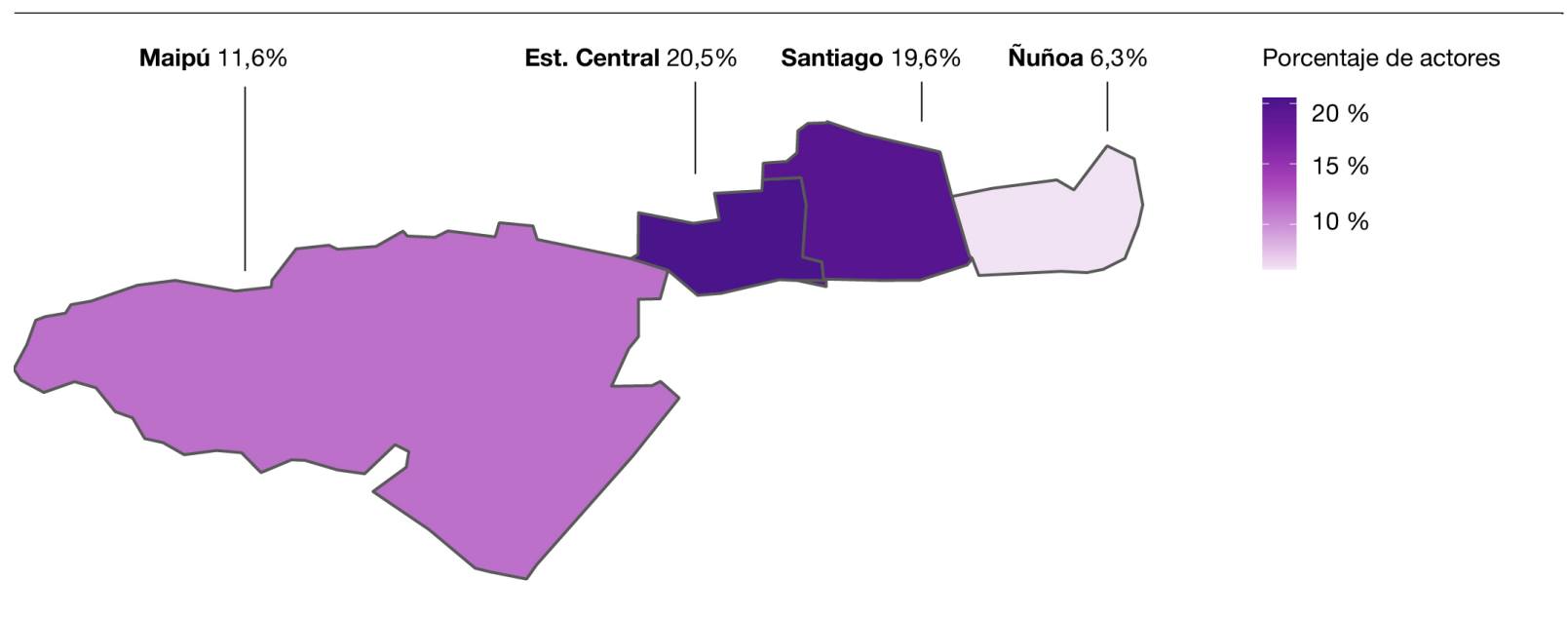

Fuente: elaboración propia desde los datos del Fondo VIME-USACH

En síntesis, la USACH, mediante su instrumento de desarrollo de proyectos de vinculación con el medio, cubre un amplio territorio de la región, y sin embargo se concentra principalmente en el entorno inmediato y en particular en tres comunas. Si bien las iniciativas no son dirigidas a impactar en el entorno inmediato a través de definiciones en la política institucional en las bases del Fondo VIME, se demuestra que espontáneamente los proyectos concentran su quehacer en el nivel más próximo de distancia del campus.

\section{Según prioridad social}

Al realizar el mismo ejercicio de análisis con el IPS es posible identificar que las comunas con una mayor prioridad en la Región Metropolitana no son necesariamente el foco de las actividades de vinculación con el medio. Esto se puede explicar si se comprende el apartado anterior, ya que las comunas del entorno inmediato -en especial las que más actividades concentran - se ubican en las categorías de media baja o baja prioridad social.

En la Figura 7 se observan las comunas en las que se ubican actores del medio y comunidades con los que se desarrollan los proyectos.

Los actores extrauniversitarios son, como se planteó, del sector Educación y Social, y se ubican principalmente en comunas de baja o media baja prioridad. Como se representa en la Figura 8, las comunas de alta o media alta prioridad son los lugares en los que menos presencia existe. Solo el $14,3 \%$ de los actores es parte de comunas con una alta o media alta prioridad. Ambas categorías son superadas incluso por el 15,2 \% de actores ubicados en comunas sin prioridad social (ver Tabla 4).

Podemos concluir en este apartado que actualmente no se trabaja con especial énfasis en territorios con un IPS alto o medio alto. Sin embargo, importante es considerar que gran parte de estas comunas se encuentra fuera de la ciudad (ver Figuras 1 y 2). 
Figura 7: Comunas donde se desarrollan proyectos según Índice de Prioridad Social

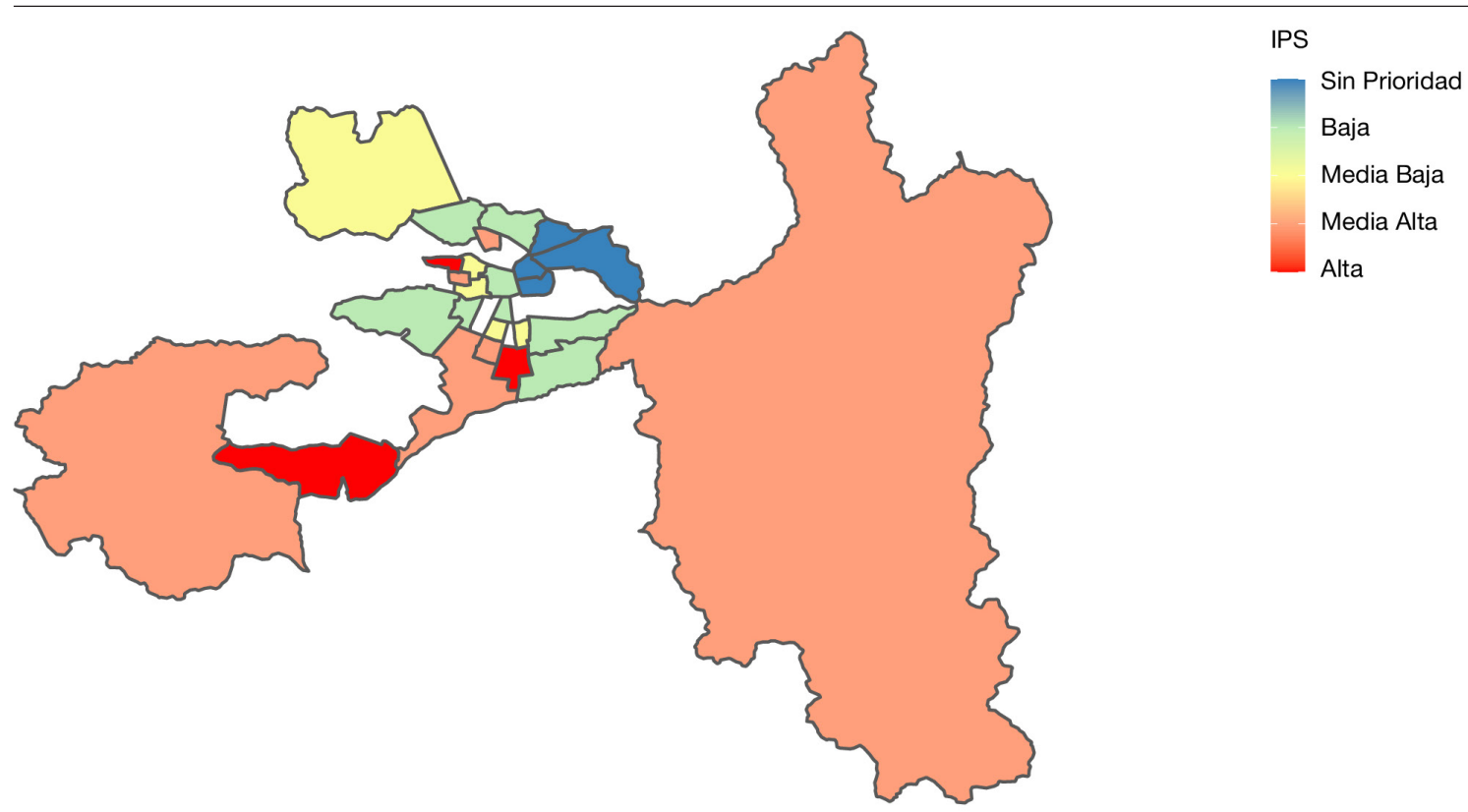

Fuente: elaboración propia según las categorías del Ministerio de Desarrollo Social y Familia (2019) y datos del Fondo VIME-USACH

Figura 8: Porcentaje de actores participantes según IPS

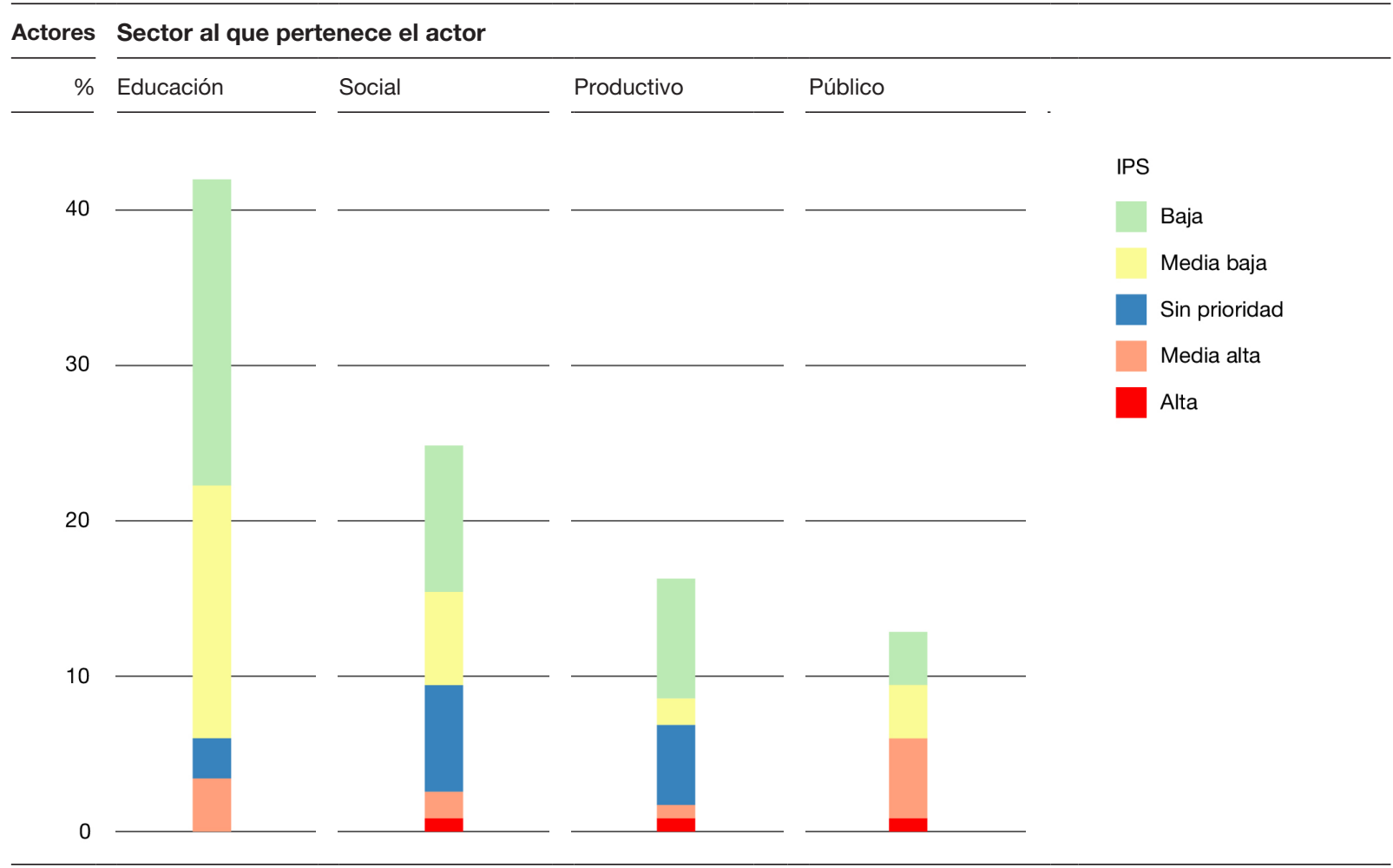

Fuente: elaboración propia según las categorías del Ministerio de Desarrollo Social y Familia (2019) y datos del Fondo VIME-USACH 
Tabla 4: porcentaje de actores extrauniversitarios según IPS por sector

\begin{tabular}{|c|c|c|c|c|c|c|}
\hline Sector & Alta & Media alta & Media baja & Baja & Sin prioridad & Total \\
\hline Educación & $0,0 \%$ & $3,6 \%$ & $17,0 \%$ & $20,5 \%$ & $2,7 \%$ & $43,8 \%$ \\
\hline Productivo & $0,9 \%$ & $0,9 \%$ & $1,8 \%$ & $8,0 \%$ & $5,4 \%$ & $17,0 \%$ \\
\hline Público & $0,9 \%$ & $5,4 \%$ & $3,6 \%$ & $3,6 \%$ & $0,0 \%$ & $13,4 \%$ \\
\hline Social & $0,9 \%$ & $1,8 \%$ & $6,2 \%$ & $9,8 \%$ & $7,1 \%$ & $25,9 \%$ \\
\hline Total & $2,7 \%$ & $11,6 \%$ & $28,6 \%$ & $42,0 \%$ & $15,2 \%$ & $100,0 \%$ \\
\hline
\end{tabular}

Fuente: elaboración propia.

\section{Conclusiones}

En la política de vinculación con el medio de la USACH se define el principio de bidireccionalidad a la hora de crear relaciones con organizaciones y actores externos. Si bien este principio debe estar presente en los proyectos adjudicados — de acuerdo con lo que se plantea en las bases de postulación-, avanzar en profundizar el estudio de este aspecto es un desafío aun pendiente, $y$ en particular se vuelve un imperativo analizar el modo en que las relaciones construidas generan dinámicas de participación sustantiva de los actores tanto en el diseño como en la ejecución y la evaluación de los proyectos.

Con relación a la política de los concursos para la obtención de financiamiento de proyectos, aun cuando se considera que es efectiva para concretar la acción de vinculación con el medio, según Tommasino y Cano, esta consolidaría "una situación de cierta fragilidad del quehacer extensionista de las universidades" (Tommasino \& Cano, 2016, p. 6). Sumado a que existe el riesgo de la realización de actividades por el mero hecho de ejecutar, en desmedro de la generación y construcción de aprendizajes, "a los cuales hay que dedicar un esfuerzo reflexivo sistemático y ordenado" (Jara, 2019, p. 5). En ese entendido, cobra relevancia que la política de financiamiento de proyectos de vinculación con el medio venga acompañada de acciones que apunten a la jerarquización de dicha función y a profundizar la integralidad, al considerar que los vínculos se generan en torno a proyectos puntuales y limitados por recursos y tiempo debido a su naturaleza misma.

Las 61 iniciativas que sirvieron de fuente de este estudio dan cuenta de que la mayoría de las organizaciones con las que se desarrollan proyectos es parte del entorno inmediato al campus universitario $\mathrm{y}$, conforme al IPS, se trabaja mayoritariamente con organizaciones de las comunas con baja prioridad social. En efecto, la USACH, mediante el Fondo VIME, construye vínculos con los actores que se hallan en el mismo territorio en el que se encuentra el campus universitario y descuida el trabajo con territorios con problemáticas sociales de más prioridad social. Un debate que deberían abrir las comunidades universitarias es si se permanece en la espontaneidad de las acciones o si se definen parámetros y criterios para priorizar los sectores a los cuales pertenecen las organizaciones o su ubicación territorial y las características de estos, dotando de un carácter estratégico la definición del medio externo sobre el cual desenvolverse.

Un tema no resuelto por completo es el desarrollo de mecanismos de medición y evaluación (Fleet et al., 2017). Aunque esta investigación busca aportar en este aspecto, es 
necesario avanzar en procesos de evaluación y sistematización de manera participativa y con las comunidades extrauniversitarias, comprendiéndolas como instancias de aprendizaje y constructoras de nuevo conocimiento. En particular, se propone integrar como criterio de medición y evaluación las siguientes aristas:

- Identificación del nivel y modo de participación de los planteles universitarios en definiciones y orientaciones para el desarrollo territorial a diversas escalas, como la barrial, comunal, regional, nacional o incluso internacional.

- El nivel de formalización e institucionalización de las instancias de diálogo con los actores extrauniversitarios, incluyéndolas en las definiciones estratégicas, plan de estudios y líneas de investigación, haciendo parte de estas definiciones las realidades territoriales.

- La manera en que son incluidos los diversos objetivos y necesidades de las comunidades y organizaciones al momento de planificar la vinculación con el medio desarrollada por las universidades.

En relación con el contexto político-social actual, cabe destacar que en lo que va de siglo la educación superior ha estado constantemente en el centro del debate público en Chile, principalmente por demandas levantadas por los movimientos sociales en torno a temas como el acceso, financiamiento, calidad y, de modo reciente, su carácter sexista. Estas tensiones son parte de un proceso movilizador que en octubre de 2019 dio origen a una crisis social sin precedentes en el último tiempo. Dicha crisis fue gatillada por el alza en el transporte público, hecho que movilizó a las y los estudiantes secundarios. Rápidamente, esa circunstancia aglutinó un sinfín de demandas y estados de ánimo que crearon las condiciones políticas y la correlación de fuerzas para un proceso de reforma constitucional, actualmente en desarrollo. Estos hechos también tensionan el quehacer universitario, con especial énfasis en el modo en que las instituciones de educación superior se relacionan con la sociedad y sus territorios.

Sumado a lo anterior, en el marco de la pandemia global actual, ${ }^{5}$ es relevante reflexionar acerca de cómo desarrollaremos la función de vinculación con el medio o extensión universitaria. Es importante tener en cuenta el riesgo de retroceder hacia un paradigma extensionista-difusionista mediante la virtualización de la función. Por otro lado, hay que considerar que existen comunidades $\sin$ acceso a las tecnologías necesarias para ello, e inclusive sin acceso a luz eléctrica las 24 horas del día en nuestros territorios. Por ende, la vía virtual no permite llegar a todos los espacios donde podrían desarrollarse instancias de vinculación.

La vinculación con el medio aparece entonces como la vía para pensar y construir universidades de nuevo tipo, en constante y vivo vínculo con la sociedad, con perspectiva de crear relaciones democratizantes y con capacidades de incidir en la transformación de la realidad.

\section{Referencias bibliográficas}

Adán, L.; Poblete, F.; Angulo, C.; Loncomilla, L. \& Muñoz, Z. (2016). La función de vinculación o tercera misión en el contexto de la educación superior chilena. Cuadernos de investigación, (1). Comisión Nacional

5) Al momento de redactar este trabajo, la Región Metropolitana y la ciudad de Santiago de Chile se encuentran en confinamiento debido a la crisis sanitaria generada por el coronavirus. 
de Acreditación. https://investigacion.cnachile.cl/archivos/cna/documentos/Cuaderno-1_Ada-An-Digital.pdf Addie, J.-P. D. (2019). Urban(izing) University Strategic Planning: An Analysis of London and New York City. Urban Affairs Review, 55(6), 1612-1645. https://doi.org/10.1177/1078087417753080

Arzeno, M. (2018). Extensión en el territorio y territorio en la extensión. Aportes a la discusión desde el campo de la Geografía. +E: Revista de Extensión Universitaria, 8(8), 3-11. https://doi.org/10.14409/extension.v8i8. Ene-Jun.7709

Bellei, C. (2020). Educación para el Siglo 21 en el Siglo 21. ¿Tomamos el tren correcto? En Corvera, M. T. \& Muñoz, G. (Eds.), Horizontes y propuestas para transformar el sistema educativo chileno (pp. 79-103). Biblioteca del Congreso Nacional. https://www.bcn.cl/publicaciones/ediciones-bcn/detalle_libro?id=10221.1/78612 Blalock, H. M. (1998). Estadística social. Fondo de Cultura Económica.

Dougnac, P. (2016). Una revisión del concepto anglosajón public engagement y su equivalencia funcional a los de extensión y vinculación con el medio. Pensamiento Educativo. Revista de Investigación Educacional Latinoamericana, 53(2), 1-19. https://doi.org/10.7764/PEL.53.1.2016.11

Fleet, N.; Victorero, P.; Lagos, F.; Montiel, B. \& Cutipa, J. (2017). Midiendo la vinculación de las instituciones de educación superior con el medio y su impacto. Estudio de las mejores prácticas en el mundo y desarrollo de instrumento piloto para instituciones chilenas. Cuadernos de investigación, (6). Comisión Nacional de Acreditación.

González, L. B.; Saravia, R. P.; Carroza, A. N.; Gasconi, M. F.; Dinamarca, N. C. \& Castro, V. L. (2017). Vinculación con el medio y territorio heterogeneidad de modelos, prácticas y sentidos en las universidades chilenas. Observatorio de Participación Social y Territorio. https://participacionsocialyterritorio.cl/web/wp-content/ uploads/2019/07/Sistematizacion-VcM-por-CTyT-y-Observatorio-UPLA.pdf

Jara, H. O. (2019). ¿Por qué y para qué sistematizar las experiencias de extensión universitaria? +E: Revista de Extensión Universitaria, 9(11), 3-9. https://doi.org/10.14409/extension.v9i11.Jul-Dic.8675

Kirberg, E. (1970). Balance del proceso reformista. En F. Rivera Tobar (2016). Enrique Kirberg: Escritos Escogidos. (pp 187-216). Ediciones de la Corporación Cultural Universidad de Santiago de Chile.

Orellana, C. V.; Canales, C. M.; Bellei, C. C. \& Guajardo, M. F. (2019). Individuación y mercado educacional en chile. Revista Brasileira de Política e Administração da Educação, 35(1), 141-157. https://doi.org/10.21573/ vol1n12019.89879

Orellana, C. V. (2016). El eco hacendal en la educación superior chilena y los desafíos de la universidad pública del siglo XXI. Anales de la Universidad de Chile, (11), 95-115. https://doi.org/10.5354/0717-8883.2017.45231

Park, R. \& Burgess, E. (1984). The City: Suggestions for Investigation of Human Behavior in the Urban Environment. The University of Chicago Press.

Programa de las Naciones Unidas para el Desarrollo (PNUD) (2018). Vinculación con el medio en las universidades estatales chilenas: Informe final.

Sousa Santos, B. de (2007). La universidad en el siglo XXI. Para una reforma democrática y emancipadora de la universidad. Plural editores.

Tommasino, H. \& Cano, A. (2016). Modelos de extensión universitaria en las universidades latinoamericanas en el siglo XXI: tendencias y controversias. Universidades, (67). 7-24. https://www.redalyc.org/ pdf/373/37344015003.pdf

Universidad de Santiago de Chile (USACH) (2018). Política de Vinculación con el Medio. Vicerrectoría de Vinculación con el Medio. https://www.vime.usach.cl/politica-vime

Vicerrectoría de Vinculación con el Medio (2019). Bases Fondo de Vinculación con el Medio. Universidad de Santiago de Chile. https://www.vime.usach.cl/sites/vime/files/paginas/bases_fondo_vime_.pdf 


\section{Fuentes}

Ley $\mathrm{N}^{\circ}$ 20129, 17 de noviembre de 2006. http://bcn.cl/2cvms

Ministerio de Desarrollo Social y Familia (2019). Región Metropolitana de Santiago. Índice de Prioridad Social de Comunas 2019. Seremi de Desarrollo Social y Familia Metropolitana. http://www.desarrollosocialyfamilia. gob.cl/storage/docs/INDICE._DE_PRIORIDAD_SOCIAL_2019.pdf 